“(C) 2021 IEEE. Personal use of this material is permitted. Permission from IEEE must be obtained for all other uses, in any current or future media, including reprinting/republishing this material for advertising or promotional purposes, creating new collective works, for resale or redistribution to servers or lists, or reuse of any copyrighted component of this work in other works." 


\section{One Dimensional Leaky-Wave Antennas with Continuous Scan of Radiating Beam}

\author{
Debabrata K. Karmokar \\ UniSA STEM \\ University of South Australia \\ Mawson Lakes, SA 5095, Australia \\ E-mail: dkkarmokar@ieee.org
}

\author{
Dushmantha N. P. Thalakotuna \\ School of Electrical and Data Engineering \\ University of Technology Sydney \\ Sydney, Australia
}

\author{
Ladislau Matekovits \\ Dipartimento di Elettronica \\ Politecnico di Torino \\ Turin, Italy
}

E-mail: Dushmantha.Thalakotuna@uts.edu.au E-mail: ladislau.matekovits@polito.it

Karu P. Esselle

School of Electrical and Data Engineering

University of Technology Sydney

Sydney, Australia

E-mail: karu@ieee.org

\begin{abstract}
Leaky-wave antennas (LWAs) have the ability to scan beam with frequency. However, the major limitation faced by most of the LWAs, particularly uniform LWAs, is their inability to scan beam through broadside. A method to achieve a continuous beam scan from backward to forward is discussed here. A substrate-integrated waveguide (SIW)-based structure is used for the study. The SIW-based LWA with only horizontal (longitudinal) slots is unable to scan the beam through the broadside due to an open stopband (OSB). The OSB is closed by using additional vertical (transverse) slots and groups of shorting vias. The simulated results show that the LWA can scan beam continuously from $-47^{\circ}$ to $+27^{\circ}$ when frequency sweeps from 8.75 to $13 \mathrm{GHz}$.
\end{abstract}

Index Terms-Leaky-wave antenna, beam scanning, broadside, continuous scanning, one-dimensional, periodic structure, substrate integrated waveguide.

\section{INTRODUCTION}

Leaky-wave antennas (LWAs) are promising candidates for future wireless systems because of their exceptional characteristics compared to other antenna structures. Among others, these include narrow beam, large bandwidth, and, more importantly, beam scanning ability with the frequency of the input signal [1]-[7]. The direction of the radiating antenna beam, from the broadside $\left(0^{\circ}\right)$, is given by $\sin ^{-1}\left[\beta_{\mathrm{n}}(\mathrm{f}) / \mathrm{k}_{0}(\mathrm{f})\right]$, where $k_{0}$ is the wavenumber in the free space. The other term of the expression, i.e., $\beta_{n}$, is the phase constant for the $n^{\text {th }}$ spatial harmonic, which is expressed in terms of the phase constant $\left(\beta_{0}\right)$ of the fundamental mode and the period $\left(L_{\text {Period }}\right)$ of the structure as $\beta_{0}+2 n / L_{P}$ eriod [8]-[10]. It is seen from the above expression that the direction of the main beam of an LWA is dispersive, i.e., frequency-dependent, since both $\beta_{n}$ and $k_{0}$ varies with the source frequency and hence the beam can be scanned by simply changing the frequency of the signal. However, one of the major limitations of an LWA, especially in the case of a uniform LWA and even many periodic LWAs, is the difficulty to scan the beam continuously from backward to forward. In most cases, it is impossible for these LWAs to even

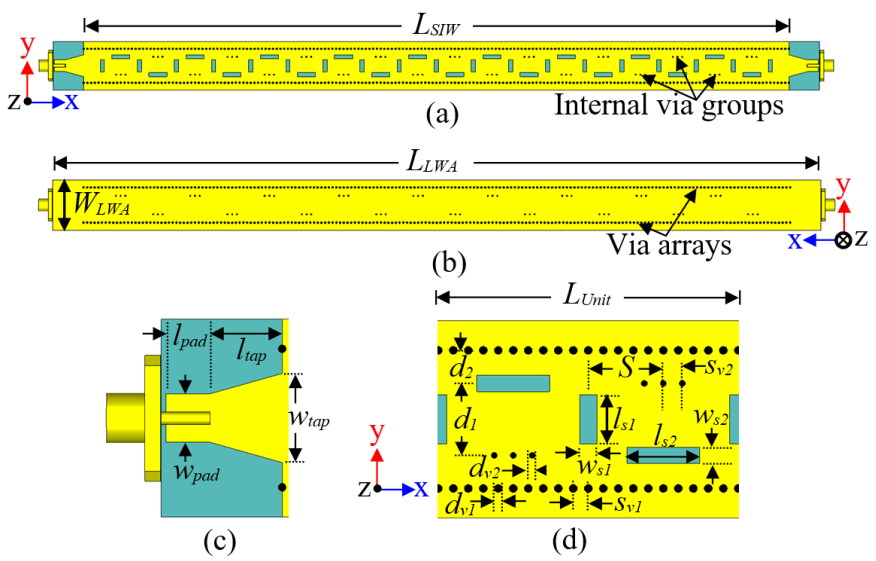

Fig. 1. Configuration of the backward to forward scanning SIW-based LWA. (a) Top view. (b) Back view. (c) Microstrip to SIW Transition. (d) Unit cell with detailed dimensions.

radiate towards the broadside direction without considerable gain loss, which is due to an open stopband that restricts radiation around that region [11], [12]. The cause of the open stopband is mainly due to the reflection of the power from the unit cells rather than radiation [10]. This limitation of LWA is an active research topic for over a decade, and different approaches were developed so far to overcome this issue. Among them, the widely used method to utilize a composite right/left-handed structure as a main radiating structure [13][18] and another popular method to use a periodic structure for such an LWA design [11], [19]-[23].

This paper addresses this challenge of open stopband and discusses a method to overcome it successfully. The stopband is closed by exciting the $n=-1$ spatial harmonic and using an impedance matching technique. Unlike the structure in [20], in which a vertical slot is used at the mid-point of the horizontal slot and two vias are used, in this structure, the vertical slots 
TABLE I

DIMENSIONS (IN MM) OF THE BACKWARD TO FORWARD SCANNING LWA IN FIG. 1, ITS UNIT CELL AND THE MICROSTRIP TO SIW TRANSITION.

\begin{tabular}{ccccccc}
\hline$L_{S I W}$ & $L_{L W A}$ & $W_{L W A}$ & $l_{\text {pad }}$ & $w_{\text {pad }}$ & $l_{\text {tap }}$ & $w_{\text {tap }}$ \\
\hline 285 & 309.8 & 20 & 4.5 & 4.9 & 7.4 & 9 \\
\hline$d_{v 1}$ & $s_{v 1}$ & $d_{v 2}$ & $s_{v 2}$ & $l_{s 1}$ & $w_{s 1}$ & $l_{s 2}$ \\
\hline 0.8 & 1.5 & 0.6 & 1.9 & 5 & 1.7 & 7.3 \\
\hline & $w_{s 2}$ & $d_{1}$ & $d_{2}$ & $S$ & $L_{\text {Unit }}$ & \\
\hline & 1.65 & 7.3 & 3.35 & 7.5 & 30 & \\
\hline \hline
\end{tabular}

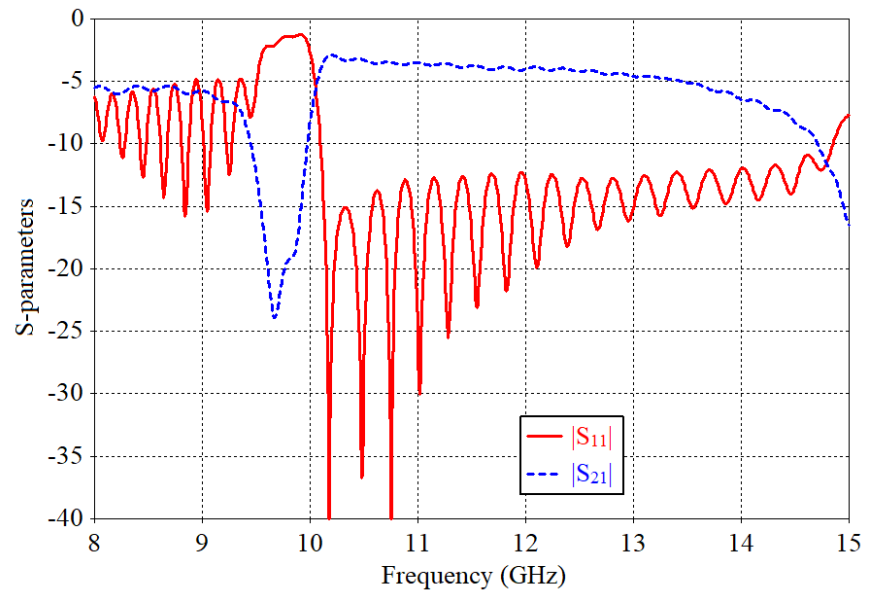

Fig. 2. Simulated S-parameters of the SIW-based LWA without the vertical slots and internal via groups.

are used between two horizontal slots and a group of three vias are used the opposite side of the horizontal slots in each unit cell. The study shows that the OSB can be closed by proper impedance matching regardless of the position of the reactive loading. Overcoming the open stopband issue increases the beam scanning range significantly. This allows such antennas to be used in applications like frequency scanning radars that require wide bandwidths and a fan beam pattern.

\section{SUPPRESSION OF THE OSB AND ACHIEVING CONTINUOUS BEAM SCAN}

As mentioned earlier, the main structure used to design the antenna is a substrate integrated waveguide. The final antenna configuration is shown in Fig. 1, consisting of nine unit cell, and the dimensions are given in Table I. When the top metal of the SIW loaded with only horizontal slots, i.e., when the vertical slots and internal via groups are absent in the structure, the antenna can still radiate, but an OSB restricts the broadside radiation. The simulated scattering parameters $\left(\left|S_{11}\right|\right.$ and $\left.\mid S_{21}\right)$ of the antenna without the vertical slots and via groups are shown in Fig. 2. It is seen from Fig. 2 that there is an OSB. Although the impedance matching can be improved by further tuning the dimensions and position of the slots, the antenna is unable to scan the beam from backward to forward without significant gain loss around the OSB region.

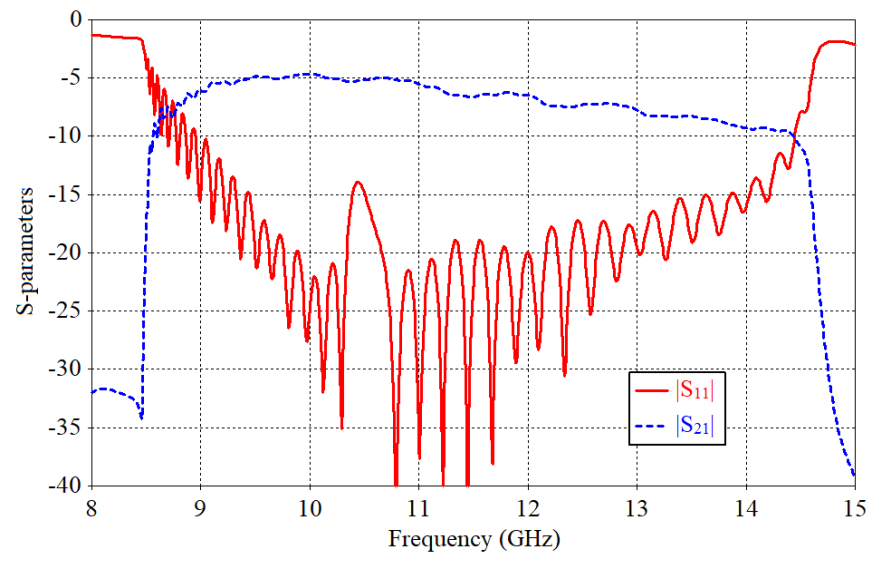

Fig. 3. Simulated S-parameters of the SIW-based LWA.

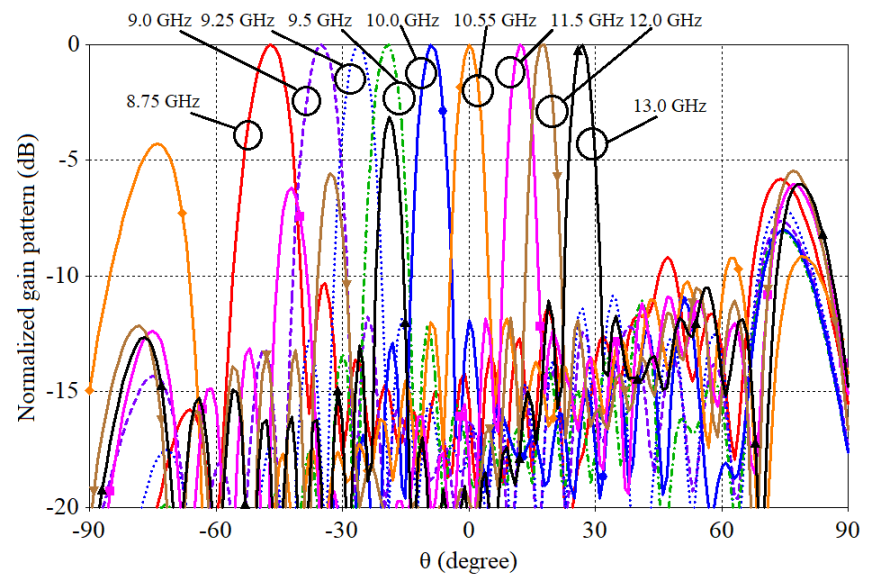

Fig. 4. Simulated normalized radiation patterns of the SIW-based LWA.

In the initial antenna, i.e. with only horizontal slots, the slots are capacitive. In order to remove the OSB, an inductive loading is required. A combination of transverse slot and a group of vias are used in each unit cell as an inductive load. The transverse slot and the group of vias help to mitigate the capacitive effect and match the impedance around the broadside frequencies, and hence the OSB suppressed. The simulated scattering parameters and normalized radiation patterns of the final antenna are shown in Fig. 3 and Fig. 4, respectively. It is seen that the $\left|S_{11}\right|$ is below $-10 \mathrm{~dB}$ within a frequency range of 8.95 to $14.44 \mathrm{GHz}$. For the frequencies around the broadside beam, the simulated reflection coefficient remains below $-13.9 \mathrm{~dB}$, indicating a good impedance matching. The value of $\left|\mathrm{S}_{21}\right|$ remains below $-4.6 \mathrm{~dB}$ throughout the impedance bandwidth, indicating good radiation performance of the antenna. From the radiation patterns, it is seen that the antenna scans continuously through the broadside and the beam points at $0^{\circ}$ at $10.55 \mathrm{GHz}$. The beam scans from $-47^{\circ}$ to $+27^{\circ}$ with a frequency sweep from 8.75 to $13 \mathrm{GHz}$. It is seen that at higher frequencies, there are additional backward beams, which usually occurs due to higher-order 


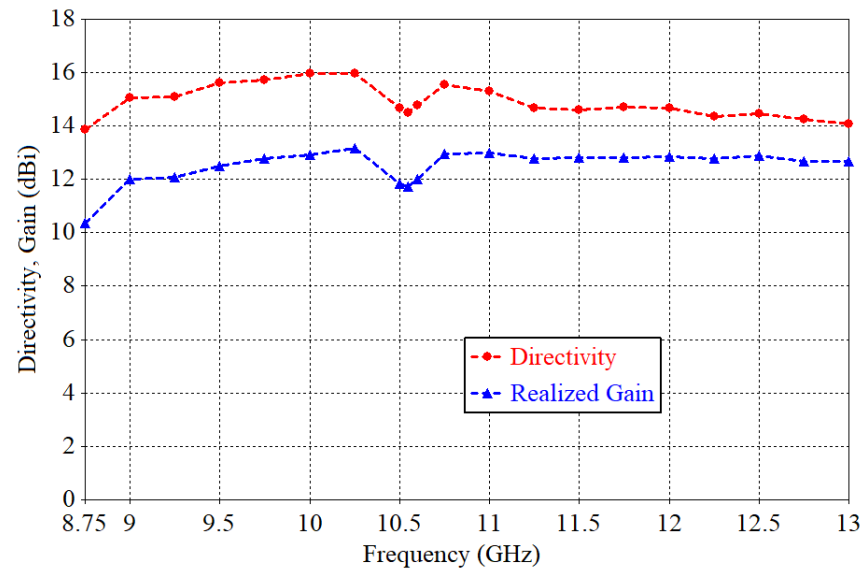

Fig. 5. Simulated gain and directivity of the SIW-based LWA.

space harmonics. To reduce the magnitude of the backward beams, the higher-order space harmonic need to be suppressed. The directivity and realized gain of the antenna is shown in Fig. 5. It is seen that the antenna has a stable gain within the beam scan range with a maximum gain of $13.17 \mathrm{dBi}$ at $10.25 \mathrm{GHz}$. The minimum antenna gain is $10.36 \mathrm{dBi}$ at $8.75 \mathrm{GHz}$, and the gain of the broadside beam is $11.73 \mathrm{dBi}$ at $10.55 \mathrm{GHz}$.

\section{CONCLUSION}

A method to achieve a continuous beam scan from backward to forward is discussed and verified using a full-wave simulation of a SIW-based LWA. The open-stopband was suppressed by using transverse slots and groups of vias. The antenna is capable of scanning the radiation beam in an angular range of $71^{\circ}$ with a gain variation of $2.81 \mathrm{~dB}$.

\section{REFERENCES}

[1] D. Zheng, Y. Lyu, and K. Wu, "Transversely slotted SIW leaky-wave antenna featuring rapid beam-scanning for millimeter-wave applications," IEEE Trans. Antennas Propag., pp. 1-1, 2020.

[2] D. K. Karmokar, K. P. Esselle, D. N. P. Thalakotuna, M. Heimlich, and L. Matekovits, "A leaky-wave antenna for beam steering in forward and backward directions," in Proc. IEEE 2013 Tencon - Spring, 2013, pp. 47-50.

[3] N. Nguyen-Trong, T. Kaufmann, L. Hall, and C. Fumeaux, "Optimization of leaky-wave antennas based on non-uniform HMSIW," in Proc. IEEE MTT-S Int. Conf. NEMO, Aug. 2015.

[4] M. Kuznetcov, V. Gomez-Guillamon Buendia, Z. Shafiq, L. Matekovits, D. Anagnostou, and S. K. Podilchak, "Printed leaky-wave antenna with aperture control using width-modulated microstrip lines and TM surfacewave feeding by SIW technology," IEEE Antennas Wireless Propag. Lett., vol. 18, no. 9, pp. 1809 - 1813, Sep. 2019.
[5] D. K. Karmokar, D. N. P. Thalakotuna, K. P. Esselle, M. Heimlich, and L. Matekovits, "Fixed-frequency beam steering from a stub-loaded microstrip leaky-wave antenna," in Proc. Int. Symp Electromagnetic Theory, 2013, pp. 436-439.

[6] M. K. Mohsen et al., "Design for radiation broadside direction using half-width microstrip leaky-wave antenna array," AEU - Int. J. Electronics Communications, vol. 110, p. 152839, 2019.

[7] D. K. Karmokar, D. N. P. Thalakotuna, K. P. Esselle, and M. Heimlich, "Controlling the beam scanning limits of a microstrip leaky-wave antenna," in 2013 IEEE Antennas and Propagation Society International Symposium (APSURSI), 2013, pp. 1330-1331.

[8] D. R. Jackson, C. Caloz, and T. Itoh, "Leaky-wave antennas," Proc. IEEE, vol. 100, no. 7, pp. 2194-2206, Jul. 2012.

[9] F. Xu, K. Wu, and X. Zhang, "Periodic leaky-wave antenna for millimeter wave applications based on substrate integrated waveguide," IEEE Trans. Antennas Propag., vol. 58, no. 2, pp. 340-347, Feb. 2010.

[10] A. A. Oliner and D. R. Jackson, "Leaky-wave antennas," in Antenna Engineering Handbook, J. L. Volakis, Ed. McGraw-Hill, 2007, ch. 11.

[11] D. K. Karmokar, Y. J. Guo, P. Y. Qin, S.-L. Chen, and T. S. Bird, "Substrate integrated waveguide-based periodic backward-to-forward scanning leaky-wave antenna with low cross-polarization," IEEE Trans. Antennas Propag., vol. 66, no. 08, pp. 3846-3856, Aug. 2018.

[12] S. K. Podilchak, L. Matekovits, A. P. Freundorfer, Y. M. M. Antar, and M. Orefice, "Controlled leaky-wave radiation from a planar configuration of width-modulated microstrip lines," IEEE Trans. Antennas Propag., vol. 61, no. 10, pp. 4957-4972, Oct. 2013.

[13] L. Liu, C. Caloz, and T. Itoh, "Dominant mode leaky-wave antenna with backfire-to-endfire scanning capability," Electron. Lett., vol. 38, no. 23, pp. 1414-1416, Nov. 2002.

[14] D. K. Karmokar, Y. J. Guo, S. Chen, and T. S. Bird, "Composite right/left-handed leaky-wave antennas for wide-angle beam scanning with flexibly chosen frequency range," IEEE Trans. Antennas Propag., vol. 68 , no. 1 , pp. 100-110, 2020.

[15] Nasimuddin, Z. N. Chen, and X. Qing, "Multilayered composite right/left-handed leaky-wave antenna with consistent gain," IEEE Trans. Antennas Propag., vol. 60, no. 11, pp. 5056-5062, Nov. 2012.

[16] _ , "Substrate integrated metamaterial-based leaky-wave antenna with improved boresight radiation bandwidth," IEEE Trans. Antennas Propag., vol. 61, no. 7, pp. 3451-3457, Jul. 2013.

[17] D. K. Karmokar, S. Chen, T. S. Bird, and Y. J. Guo, "Single-layer multivia loaded CRLH leaky-wave antennas for wide-angle beam scanning with consistent gain," IEEE Antennas Wireless Propag. Lett., vol. 18, no. 2, pp. 313-317, Feb. 2019.

[18] H. Jiang et al., "Backward-to-forward wide-angle fast beam-scanning leaky-wave antenna with consistent gain," IEEE Trans. Antennas Propag., pp. 1-1, 2020.

[19] R. Ranjan and J. Ghosh, "SIW-based leaky-wave antenna supporting wide range of beam scanning through broadside," IEEE Antennas and Wireless Propagation Letters, vol. 18, no. 4, pp. 606-610, Apr. 2019.

[20] D. K. Karmokar, S. Chen, D. Thalakotuna, P. Qin, T. S. Bird, and Y. J. Guo, "Continuous backward-to-forward scanning 1-D slot-array leakywave antenna with improved gain," IEEE Antennas Wireless Propag. Lett., vol. 19, no. 1, pp. 89-93, 2020.

[21] S.-L. Chen et. al., "Polarization-reconfigurable leaky-wave antenna with continuous beam scanning through broadside," IEEE Trans. Antennas Propag., vol. 68, no. 1, pp. 121-133, 2020.

[22] A. K. Tiwari, S. Awasthi, and R. K. Singh, "A symmetrical periodic leaky-wave antenna with backward-to-forward scanning," IEEE Antennas and Wireless Propagation Letters, vol. 19, no. 4, pp. 646-650, 2020.

[23] P. Burghignoli, G. Lovat, and D. R. Jackson, "Analysis and optimization of leaky-wave radiation at broadside from a class of 1-D periodic structures," IEEE Trans. Antennas Propag., vol. 54, no. 9, pp. 2593 2604, Sep. 2006. 